\title{
Transcranial Magnetic Stimulation in Psychiatric Research
}

\author{
B. K. PURI and S.W. LEWS
}

The recording and application of electrical energy to the human brain for research, diagnostic and therapeutic purposes has been closely associated with psychiatry during this century. Berger first recorded electrical activity from the human brain in 1929, Cerletti and Bini published their results of the use of electroconvulsive therapy (ECT) in 1938, and the field of evoked potentials has continued to progress since the introduction of signal averaging by Dawson in 1949. While it is well known that electricity and magnetism are closely related, it has only been in the last decade or so that technical advances have allowed the possibility of analogous uses of magnetism in psychiatry. The development of the superconducting quantum interference device (SQUID) has made practical the recording of the magnetoencephalogram, while transcranial magnetic stimulation (TMS) has been introduced as a method for safely and painlessly stimulating the cerebral cortex through the intact skull using a coil delivering a rapidly changing magnetic field (Barker et al, 1985). Are there emerging possibilities of the use of TMS for research, diagnostic and even therapeutic purposes in psychiatry?

\section{History}

Exactly 100 years ago d'Arsonval first demonstrated, by passing electrical currents through a coil surrounding the head, that a changing magnetic field can stimulate excitable human tissue. Effects he noted included vertigo, occasional syncope, bright spots in the visual field known as phosphenes, and muscular contraction. The report of phosphenes suggests there was stimulation of the retina or visual cortex, while the muscular contractions probably resulted from stimulation of the motor cortex. Magnetically induced phosphenes continued to be studied during the early years of this century.

Little further work was carried out on the effects of large time-varying magnetic fields for over half a century. Barker (1976) studied the feasibility of using a magnetic field pulse to stimulate nerves in humans, with the aim of recording resulting nerve and muscle action potentials. In 1985 his group in Sheffield, England, managed to produce twitches in arm and leg muscles, without causing distress or pain, using a flat $10 \mathrm{~cm}$ diameter coil on the intact scalp (Barker et al, 1985), over the region of the motor cortex.

\section{How does it work?}

TMS has been found to be safe and painless. The current induced in the brain is about 1/100 000 the size of the inducing current. The energy used with TMS is around a million times smaller than that used for a stimulus delivered with ECT, with the total energy delivered to the brain to cause depolarisation typically being around $0.1 \%$ of the basal metabolic rate of the brain itself (Cadwell, 1990). The amount of energy imparted by standard TMS to small metallic objects that may be found in the head, such as aneurysm clips, is exceedingly small and does not pose a danger (Cadwell, 1990).

The site of action of TMS in cortical areas such as the motor cortex remains a matter of controversy. Different investigators have argued either that the electric field induced in the brain activates corticospinal cells directly, with activation taking place at or very close to the initial segment of the neurons (Edgley et al, 1990; Baker et al, 1994), or that instead intracortical elements are first activated and that these subsequently discharge the cortico-motoneuronal cells trans-synaptically (Day et al, 1989).

\section{TMS as a diagnostic or therapeutic tool}

TMS has begun to be used diagnostically in the management of conversion disorder. For example, Jellinek et al (1992) described a case of presumed hysterical paraplegia precipitated by spinal injury in a patient with a previous history of surgery for scoliosis. Motor evoked potentials were elicited with TMS 12 days after injury and were within normal limits. The presence of normal motor 
electrophysiology and the observation by the patient of involuntary movement of his lower limbs during TMS clinched the diagnosis and facilitated his management.

It has also been suggested that repetitive TMS (rTMS) could provide a safer alternative to ECT. In the first report of such a therapeutic use, Greenberg et al (1995) administered rTMS, on different days and over different prefrontal sites, to clinically depressed patients, patients with obsessive-compulsive disorder (OCD), and normal controls. Preliminary evidence suggested that left prefrontal rTMS could improve depression but at some stimulus parameters induce sadness in normal controls. Right prefrontal rTMS could worsen depression in the depressed patients and worsen anxiety in some OCD patients. More work is needed.

\section{TMS as a research tool}

We have begun to look at the feasibility and possible usefulness of TMS in schizophrenia. In the first study of motor function in schizophrenia using TMS, the latency of compound motor evoked potentials following TMS applied to the motor cortex was found to be significantly shorter in schizophrenic patients compared with normal controls (Puri et al, 1996a, in. this issue). This finding was not predicted and needs replicating. However, it is consistent with any of the following possible abnormalities in schizophrenia: a relative lack of corticospinal inhibition of motor responses; a change in the site of TMS activation; or an abnormality of peripheral nervous function. Drug effects were unlikely to be the cause since the patients were drug-free, most being drug-naive. Following treatment, the latency of electromyographic suppression was significantly longer in patients taking antipsychotic medication than in untreated patients (Puri et al, 1996b).

It can be argued that TMS also has a potentially important role in complementing functional imaging studies in psychiatry. For example, in studies of affective disorders, demonstrating an association between regional cerebral activity and a transient emotional state does not necessarily imply that the specific brain region and emotional state are causally linked (Goldman-Rakic, 1988). Thus, in discussing the results of their positron emission tomography study of cerebral activity during transient sadness and happiness in healthy women, George et al (1995) acknowledge that the brain regions they demonstrated as active during transient sadness might be epiphenomenally activated and might not actually serve a direct role in mood regulation; for example, one might be more attentive during a sad state and activate, indirectly, brain regions involved in attention. They suggest the need for studies using TMS which could cause 'temporary lesions'; the effects on emotional state could then be measured, thereby implying a tighter causal connection.

At present, TMS is an intriguing technique whose use in psychiatric research has yet to become clear. In that it can elicit regionally-specific, predictable, functional brain activity, one possible role immediately suggests itself. Imaging with PET and increasingly functional $M R$ and magnetoencephalography show abnormalities in linked systems of cortical activity in disorders like schizophrenia. These abnormalities seem to parallel symptom clusters such as hallucinations, or specific cognitive impairments. Intriguing as this research is, the interpretations of the findings are often post hoc: that is, functional abnormalities are detected in the absence of a prior hypothesis. It is sometimes difficult to evaluate such studies. TMS might offer a second-stage test of these hypotheses by stimulating those cortical regions implicated in symptom production in patient volunteers. If the regions are indeed involved in symptom production then stimulating them with TMS might alter transiently the subjective experience of the symptoms in a replicable way. For instance, if subjects prone to hallucinations have altered activity in supplementary motor area and middle temporal gyrus (McGuire et al, 1995) can TMS over these regions switch on (or off) hallucinations where TMS at other regions fails to do so?

\section{Roferences}

BAKar, S. N., Ouvier, E. \& LeMON, R. N. (1994) Recording an identified pyramidal volley evoked by transcranial magnetic stimulation in a conscious macaque monkey. Experimental Brain Research, 99, 529-532.

BARKER, A. T. (1976) Determination of the distribution of conduction velocities in human nerve trunks. $\mathrm{PhD}$ thesis. Sheffield: University of Sheficield.

- Jalnous, R. \& Freeston, I. L. (1985) Non-invasive magnetic stimulation of human motor cortex (letter). Lancet, 1 , 1106-1107.

CADWELL, J. (1990) Principles of magnetoelectric stimulation. In Magnetic Stmulation in Clinical Neurophysiology (ed. S. Chokroverty), pp. 13-32. Boston: Butterworths.

Day, B. L., Dressler, D., Makrtens de NoOrdhout, A., et al (1989) Electric and magnetic stimulation of human motor cortex: surface EMG and single motor unit responses. Journal of Physiology, 412, 449-473.

Edoley, S. A., EYre, J. A., Lemon, R. N., et al (1990) Excitation of the corticospinal tract by electromagnetic and electrical 
stimulation of the scalp in the macaque monkey. Journal of Physiology, 425, 301-320.

George, M. S., Ketter, T. A., Parekh, P. I., et al (1995)

Brain activity during transient sadness and happiness in healthy women. American Journal of Psychiatry, 152, 341351.

Greenberg, B. D., George, M. S., WassermanN, E. N., et al (1995) Repetitive transcranial magnetic stimulation (rTMS) of prefrontal cortex: mood and anxiety effects in health and disease (abstract). Journal of Neuropsychiatry and Clinical Neurosciences, 7, P15.

GoldmaN-RAKIC, P. S. (1988) Topography of cognition: parallel distributed networks in primate association cortex. Annual Review of Neuroscience, 11, 137-156.
Jellinek, D. A., Bradford, R., Bailey, I., et al (1992) The role of motor evoked potentials in the management of hysterical paraplegia: case report. Paraplegia, 30, 300-302.

MCGuire, P. K., Silbersweig, D. A., Wright, I., et al (1995) Abnormal monitoring of inner speech: a physiological basis for auditory hallucinations. Lancet, 346, 596-599.

Puri, B. K., Davey, N. J., Ellaway, P. H., et al (1996a) An investigation of motor function in schizophrenia using transcranial magnetic stimulation of the motor cortex. British Journal of Psychiatry, 169, 690-695.

_ LEwIS, H. S., et al (1996b) Electromyographic responses of thenar muscles to transcranial magnetic stimulation of the motor cortex in schizophrenia [abstract]. Schizophrenia Research, 18, 234-235.

B. K. Puri, MRCPsych, Robert Steiner MRI Unit, Hammersmith Hospital; S. W. Lewis, MRCPsych, School of Psychiatry and Behavioural Sciences, Department of Psychiatry, Withington Hospital, West Didsbury, Manchester M20 8LR

Correspondence: Dr B. K. Puri, Robert Steiner MRI Unit, Royal Postgraduate Medical School, Hammersmith Hospital, Du Cane Road, London W12 0NN

(Received 24 June 1996, accepted 27 June 1996) 